\title{
Facet Engineering in Metal Organic Frameworks to Improve Their Electrochemical Activity for Water Oxidation
}

Received 00th January 20xx, Accepted 00th January 20xx

DOI: $10.1039 / \times 0 \times x 00000 x$

\author{
Jiawei Wan a,e,f , Di Liu b,e, Hai Xiao ${ }^{\text {a,* }}$, Hongpan Rong c,*, Guan Sheng d, Feng Xie ${ }^{d}$, Dingsheng \\ Wang ${ }^{\mathrm{a}, *}$, and Yadong $\mathrm{Li}^{\mathrm{a}}$
}

www.rsc.org/

The facet effect has been well demonstrated for nanocrystal catalysis, however, is rarely revealed on metal organic frameworks (MOFs). Herein, we present a facile way to construct 2D zeolitic imidazolate framework-67 (ZIF-67) with dominant exposure of (002) facets. We discovered that ZIF-67 (002) facet exhibited the highest catalytic activity for oxygen evolution reaction (OER) among (002), (011), and (111) facets. Moreover, this facet engineering is also available for ZIF-8 nanocrystals.

The facet engineering has become an important strategy for well-tuning the catalytic properties in nanocrystal catalysis, ranging from heterogeneous catalysis to electrochemical catalysis, photocatalysis, and gas sensors. ${ }^{1-4}$ Because the catalytic properties such as activity, selectivity, and stability can be directly determined by the surface electronic structures, surface electron transport characteristics, and active center structures of catalysts, which can be finely tailored by surface facet control.

Metal organic frameworks (MOFs) are a class of periodic and porous micro-/nano-structured materials since they were first introduced in 1990s. ${ }^{5}$ Since the abundant metal active sites and rich pore structures within frameworks, ${ }^{6}$ they have exhibited outstanding performance in catalysis, ${ }^{7-11}$ lithiumsulfur batteries, ${ }^{12}$ gas storages, ${ }^{13}$ gas separations, ${ }^{14-15}$ and sensors ${ }^{16}$. Recently, more efforts have also been focused on the large-scale production and industrial applications of

\footnotetext{
a. Department of Chemistry, Tsinghua University, Beijing 100084, China.

b. State Key Laboratory of Inorganic Synthesis \& Preparative Chemistry, College of Chemistry, Jilin University, Changchun, Jilin 130012, China.

Beijing Key Laboratory of Construction-Tailorable Advanced Functional Materials and Green Applications, School of Materials Science \& Engineering, Beijing Institute of Technology, Beijing 100081, China.

d. Advanced Membranes and Porous Materials Center, Physical Sciences and Engineering Division, King Abdullah University of Science and Technology, Thuwal, 23955-6900, Saudi Arabia.

e. These authors contributed equally to this paper.

f. Present address: State Key Laboratory of Biochemical Engineering, Institute of Process Engineering, Chinese Academy of Sciences, Beijing 100190, P. R. China

E-mail:rhp@bit.edu.cn (H.P. Rong), haixiao@mail.tsinghua.edu.cn(H.Xiao), wangdingsheng@mail.tsinghua.edu.cn (D.S. Wang)

+ Electronic supplementary information (ESI) available: Experimental procedures and data (Fig. S1-S19, Table S1-S2). See DOI: 10.1039/x0xx00000x
}

MOFs. ${ }^{17}$ Shaping MOFs into $1-, 2-$ or 3 -dimensional forms is considered as an ideal approach to preserve or improve their special properties in practical applications ${ }^{18-22}$. However, the facet engineering in MOFs is rarely revealed.

Herein, we presented a new concept to realize the facet engineering in MOFs by shaping zeolitic imidazolate frameworks (ZIF-67) into 2D structures with exposed (002) facets. And we discovered that (002) facets of ZIF-67 exhibited the best activity for oxygen evolution reaction (OER) among (002), (011), and (111) facets. By contrast, size-comparable ZIF67 nanocrystals with mainly exposed (011) facets were found badly active for OER. By experimental and density functional theory (DFT) results, we have revealed this facet effect on OER properties of ZIF-67. Moreover, this synthetic strategy is industrially favourable due to its environmental friendly, rapid, and high-yield production.

ZIF-67 (002) was synthesized by a solution process (Fig. S1). Transmission electron microscopy (TEM) images (Fig. 1a and 1b) displayed the uniform $2 \mathrm{D}$ structures of the products. The lateral size for the 2D structures was $1.979 \pm 0.216 \mu \mathrm{m}$ (Fig. S2a). By X-ray diffraction (XRD) patterns in Fig. 1c, 2 theta values at (011), (002), (112), (013), and (114) facets of the 2D products matched well with the simulated bulk-phase ZIF-67, indicating the same ZIF-67 crystal structure of the 2D products. Interestingly, among all detected facets, the relative peak intensity of (002) in 2D ZIF-67 was the strongest one. In addition, the relative peak intensity of (011) in 2D ZIF-67 became weaker. Further, the drift-corrected high-resolution TEM (HRTEM) image of 2D ZIF-67 (Fig. 1d) and the ABSFfiltered image taken along [001] axis (inset of Fig. 1d) indicated the (002) of the exposed facet in 2D ZIF-67. Brunner-EmmetTeller (BET) measurement was performed to prove the microporous structure of the 2D ZIF-67 (002) and the BET surface area was $1731 \mathrm{~m}^{2} / \mathrm{g}$ (Fig. S3). The corresponding pore diameter size was $0.62 \mathrm{~nm}$. The scan electron microscopy (SEM) image displayed a large-scale observation and Tyndall effect of 2D ZIF-67 (002) colloidal solution proved the well dispersed aqueous solution (inset of Fig. S4). 


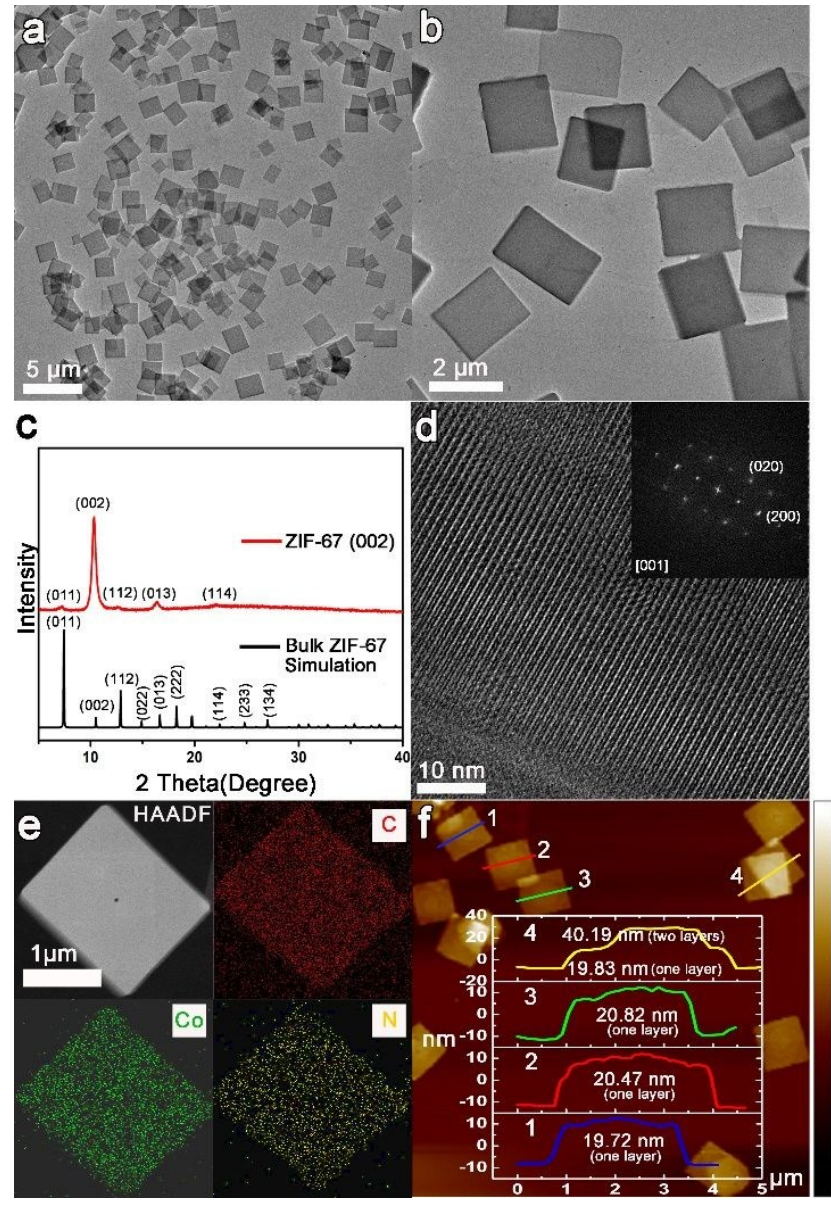

Fig. 1 (a) TEM images of ZIF-67 (002). (b) TEM images of ZIF-67 (002) in different magnification. (c) XRD patterns of ZIF-67 (002) and simulated bulk ZIF-67. (d) Drift-corrected HRTEM image of ZIF-67 (002) and ABSF-filtered image taken along [001] axis. (e) Elemental mapping of ZIF-67 (002), showing the distribution of $C$ (red), Co (green), $\mathrm{N}$ (yellow). (f) AFM results of ZIF-67 (002).

The elemental mappings illustrated the uniform distributions of $\mathrm{C}, \mathrm{N}$, and Co over the entire architecture of the 2D structures (Fig. 1e). Also, the line scan results (Fig. S5) indicated the uniform longitudinal distribution of $\mathrm{C}, \mathrm{N}$, and $\mathrm{Co}$ over the entire architecture. Energy-dispersive $\mathrm{X}$-ray spectroscopy (EDX) results showed the atomic ratios of different elements in 2D ZIF-67 (002) (Fig. S6). In addition, atomic force microscopic (AFM) measurement was performed to investigate the thickness of ZIF-67 (002) (Fig. 1f) and to give an average thickness of $20.42 \pm 0.72 \mathrm{~nm}$ (Fig. S2b).

To investigate the synthetic procedure of ZIF-67 (002), the adsorption energies of sodium dodecyl sulfate (SDS) on three typical low-index facets of (002), (011) and (111) of ZIF-67 were estimated by DFT calculations, where the SDS was approximated by $\mathrm{CH}_{3} \mathrm{SO}_{3} \mathrm{H}$ for the compromise with computational cost. We found that both (002) and (111) surfaces of $\mathrm{ZIF}-67$ bind $\mathrm{CH}_{3} \mathrm{SO}_{3} \mathrm{H}$ strongly with adsorption energies of 0.43 and $0.48 \mathrm{eV}$, respectively, while the (011) surface bind $\mathrm{CH}_{3} \mathrm{SO}_{3} \mathrm{H}$ weakly with an adsorption energy of $0.25 \mathrm{eV}$ (Table S1). Additionally, the stability of surface site contributed here: it costed 0.97, 0.96 and 0.98 evever isite ito expose (002), (011) and (111) bare Dsurfaces 3sitec Cwithout adsorption of SDS, respectively. But with adsorption of $\mathrm{CH}_{3} \mathrm{SO}_{3} \mathrm{H}$ (at a coverage of $1 / 8$ ), it costed $0.91,0.93$ and 0.94 eV per site to expose (002), (011) and (111) surface site (Table S1), respectively. Thus, the (002) facet in ZIF-67 became the most stable upon the adsorption of $\mathrm{CH}_{3} \mathrm{SO}_{3} \mathrm{H}$, which indicated the most stable of (002) facet in ZIF-67 upon the adsorption of SDS.

The formation of ZIF-67 (002) contained steps of vesicle micelle formation, assembly, and crystal growth (Fig. S7a). The transient states during the synthetic process of ZIF-67 (002) were investigated by the cryogenic scanning electron microscope (cryo-SEM) (Fig. S7b-e) and dynamic light scattering (DLS) (Fig. S8).

To verify the facet effect on catalytic properties of ZIF-67 (002), the OER was performed as the probe reaction for different samples. The ZIF-67 nanocrystals with an average size of about $56 \mathrm{~nm}$ (Fig. S9) were produced as reference catalyst, which displayed the dominant exposure of (011) facet among all detected facets (Fig. S10). The BET surface area of ZIF-67 nanocrystal was $1763 \mathrm{~m}^{2} / \mathrm{g}$ (Fig. S11).

Linear sweep voltammetry (LSV) method was performed in a standard three-electrode system (1.0 $\mathrm{M} \mathrm{KOH}$ solution) from 0 to $1.0 \mathrm{~V}$ at a scan rate of $10 \mathrm{mV} \mathrm{s}^{-1}$ (Fig. 2a). In detail, when the thermodynamic OER potential $\left(\mathrm{E}^{0} \mathrm{H}_{2} \mathrm{O} / \mathrm{O}_{2}=1.23 \mathrm{~V}\right)$ was used as the reference ${ }^{23-26}$, the ZIF-67 nanocrystals displayed an over potential of $360 \mathrm{mV}$ at the current density of $10 \mathrm{~mA} / \mathrm{cm}^{2}$. Meanwhile, 2D ZIF-67 (002) exhibited an over potential of 305 $\mathrm{mV}$ at the current density of $10 \mathrm{~mA} / \mathrm{cm}^{2}$, which was much smaller than $360 \mathrm{mV}$ of ZIF-67 nanocrystal (Fig. 2b). By contrast, the commercial $\mathrm{IrO}_{2}$ displayed an over potential of $330 \mathrm{mV}$ at $10 \mathrm{~mA} / \mathrm{cm}^{2}$. Interestingly, the 2D ZIF-67 (002) catalysts demonstrated much better OER catalytic performance than that of ZIF-67 nanocrystal. We further investigated the current density of different catalysts at the same potential of $1.61 \mathrm{~V}$ vs. RHE. The 2D ZIF-67 (002) delivered $100 \mathrm{~mA} / \mathrm{cm}^{2}$ at $1.61 \mathrm{~V}$ vs. RHE which was greatly larger than $14.78 \mathrm{~mA} / \mathrm{cm}^{2}$ of the ZIF-67 nanocrystal and $33.02 \mathrm{~mA} / \mathrm{cm}^{2}$ of the commercial $\mathrm{IrO}_{2}$. We normalized the current density at $1.61 \mathrm{~V}$ vs. RHE of catalysts by BET surface area, giving the same conclusion as above (Fig. S12). The catalytic kinetics of catalysts were investigated by Tafel plots, which were recorded with the linear regions, and the subsequently fitted via the Tafel equation $\left(\eta=b^{*} \log j+a\right.$, where $\eta$ is the overpotential, $j$ is the current density, and $b$ is the Tafel slope $)^{27}$. The ZIF-67 (002) presented a Tafel slope of $67 \mathrm{mVdec}^{-}$ 1 , which was smaller than ZIF- 67 nanocrystal $\left(79 \mathrm{mVdec}^{-1}\right)$ and the commercial $\mathrm{IrO}_{2}\left(71 \mathrm{mVdec}^{-1}\right)$, indicating a better kinetics of the ZIF-67 (002) than those of the ZIF-67 nanocrystal and the commercial $\mathrm{IrO}_{2}$ for OER (Fig. 2c). ${ }^{28}$ In addition, the residual SDS on the catalyst surface was proved no positive effect on the OER performance (Fig. S13). All above discussions indicated a much better OER performance of 2D ZIF-67 (002) than ZIF-67 nanocrystals. In addition, the 2D ZIF-67 (002) displayed a good stability for $8 \mathrm{~h}$ chronoamperometry (CA) test with only $7.2 \%$ loss of current density (Fig. $2 \mathrm{~d}$ ). The spent ZIF- 
67 (002) catalysts maintained the 2D structure (Fig. S14). XRD pattern of the spent ZIF-67 (002) still displayed the ZIF-67 crystal structure with a dominant exposure of (002) facet (Fig. S15).
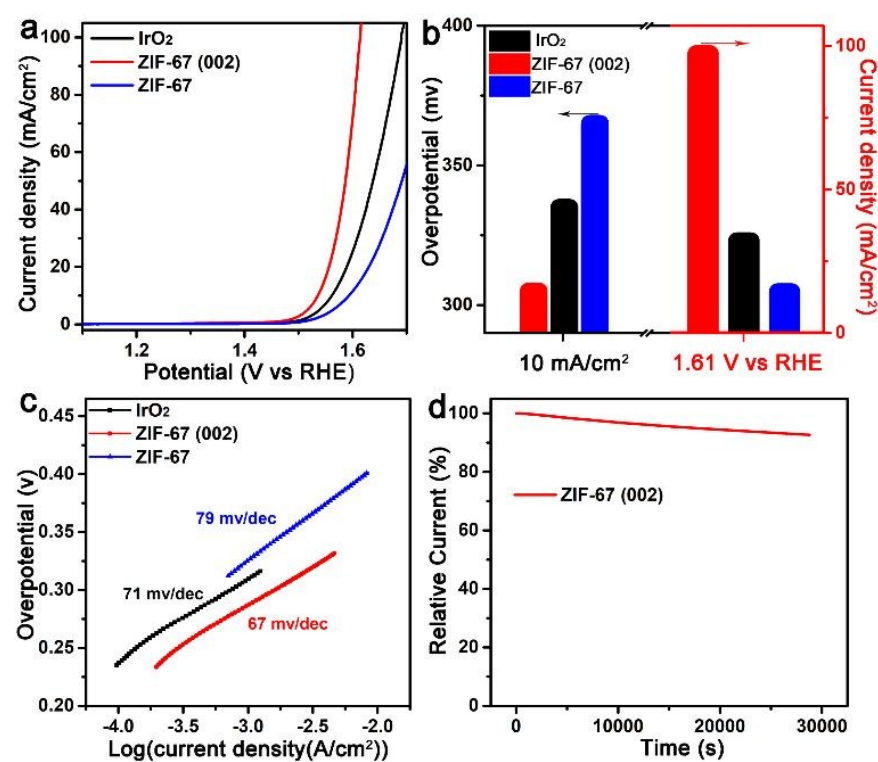

Fig. 2 (a) Linear sweep voltammetry plots of commercial IrO ${ }_{2}$, ZIF-67 (002) and ZIF-67. (b) Overpotentials and current densities of different catalysts at $10 \mathrm{~mA} / \mathrm{cm}^{2}$ and $1.61 \mathrm{~V}$ vs RHE, respectively. (c) Tafel plots of different catalysts. (d) Chronoamperometry plot of ZIF-67 (002).

Why the 2D ZIF-67 (002) displayed much better catalytic activity than that of ZIF-67 nanocrystals in OER? To answer the question and deeply understand the reaction mechanism of $2 \mathrm{D}$ ZIF-67 (002) in OER, DFT calculations were performed as the previous report ${ }^{29}$. Although the average absolute errors of DFT methods is around $0.2 \mathrm{eV}$, DFT calculations have been shown extensively to give reliable qualitative predictions, particularly for the trend of the performance of catalysts ${ }^{29-30}$. Considering the charge transfer of four electrons in the free energy profiles for OER process, we discussed the following four reactions:

${ }^{*}+\mathrm{OH}^{-} \rightarrow * \mathrm{OH}+\mathrm{e}^{-}(\mathrm{i})$

$* \mathrm{OH}+\mathrm{OH}-\rightarrow * \mathrm{O} \cdot+\mathrm{H}_{2} \mathrm{O}+\mathrm{e}^{-}(\mathrm{ii})$

$* \mathrm{O} \cdot+\mathrm{OH}^{-} \rightarrow * \mathrm{OOH}+\mathrm{e}^{-}$(iii)

$* \mathrm{OOH} \rightarrow *+\mathrm{O}_{2}+\mathrm{H}^{+}+\mathrm{e}^{-}$(iv)

where * denotes the adsorption site (surface Co site), *O-stands for the adsorbed $\mathrm{O}$ species.

As for ZIF-67 (002) in Fig. 3, we calculated the free energies of each steps. Notice that the lower limit is determined by the equilibrium potential for the overall reaction, which in this case is $1.23 \mathrm{~V}$. The largest free energy difference of $1.71 \mathrm{eV}$ was calculated for the process iii among the four, which means that at the potentials above $1.71 \mathrm{eV}$ all steps of OER are exothermic and can generally happen. The overpotential versus the RHE was obtained by the largest free energy (1.71 $\mathrm{eV})$ minus the equilibrium potential of oxygen evolution (1.23 $\mathrm{eV})$. Therefore, an overpotential of $0.48 \mathrm{~V}(\mathrm{~V}$ vs. RHE) is necessary to make all steps down-hill in free energy. The DFT calculations on (011) (Fig. S16) and (111) (Fig. S17) facetets with

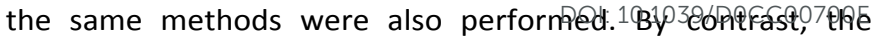
predicted overpotentials were calculated to be $0.50 \mathrm{~V}(\mathrm{~V}$ vs. RHE) and 0.49 V (V vs. RHE) for (011) and (111) facets in ZIF-67, respectively (Fig. $3 \mathrm{~b}$ and Table S2), which were larger than 0.48 $\mathrm{V}(\mathrm{V}$ vs. RHE) of (002) facets. Therefore, the better activity of (002) facet than (011) and (111) facets was proved. That's why the 2D ZIF-67 (002) demonstrated much better catalytic performance than that of the ZIF-67 nanocrystals.
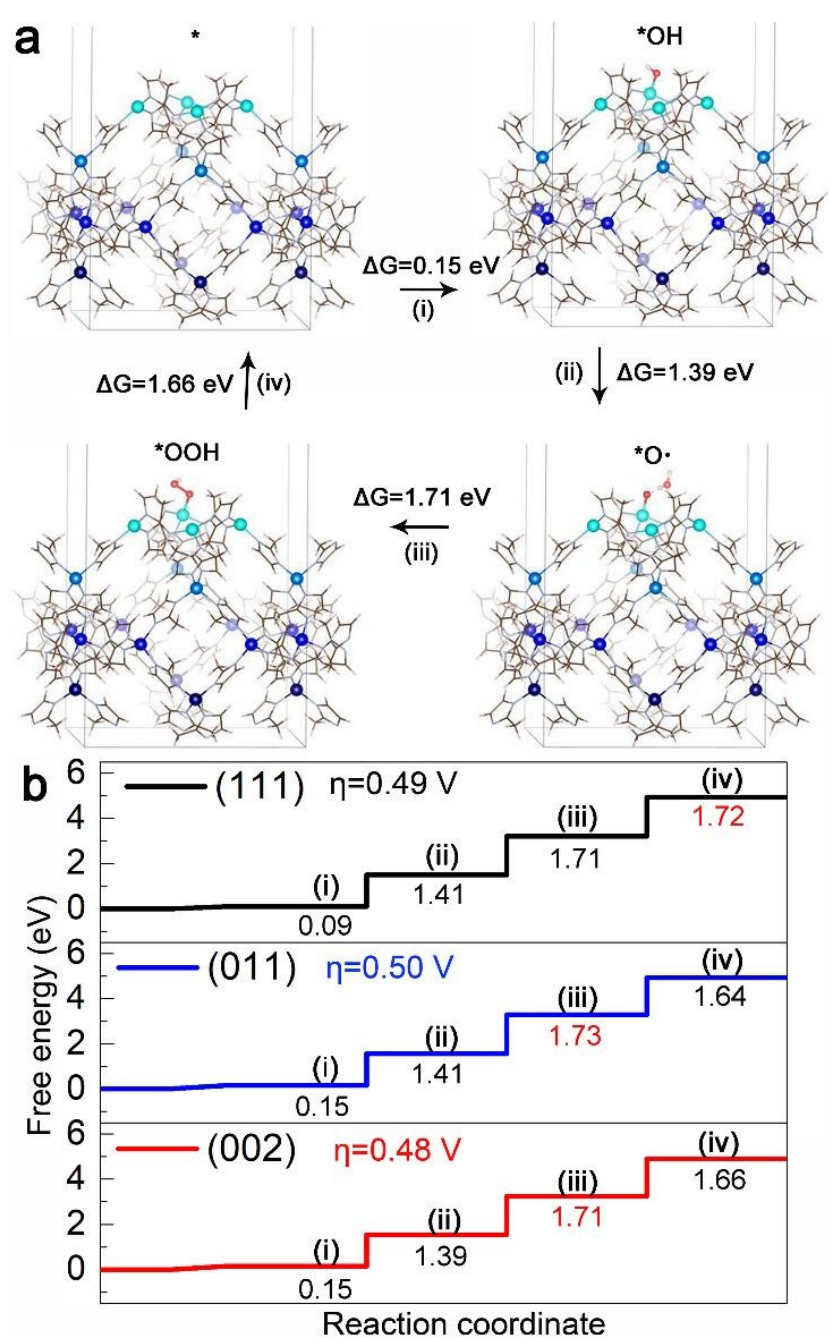

Fig. 3 (a) Energetics steps of the OER process on ZIF-67 (002) surfaces. Color scheme for chemical representation: Co atoms are colored by layers, blue for metal $\mathrm{Co}$, red for $\mathrm{O}$, and white for $\mathrm{H}$. (b) Standard free energy diagrams of the OER process on (002), (011), and (111) facets, respectively.

In addition, the production yield of ZIF-67 (002) was $~ 67.8 \%$ (Fig. S18) and its large-scale production could be easily achieved (Fig. S19 and S20). This method is also available for zinc-based ZIF-8 (002) nanocrystals (Fig. 4 and Fig. S21). Fig. 4a illustrated the 2D structure of ZIF- 8 and the inset indicated the Tyndall effect of ZIF-8 colloidal solution. $\mathrm{C}, \mathrm{N}$, and $\mathrm{Zn}$ elements 
uniformly distributed in overall the ZIF-8 (Fig. 4b). The colour picture of ZIF-8 nanosheets at large-scale was displayed in Figure 4c. The XRD pattern (Fig. 4d) indicated the ZIF-8 crystal structure with a dominant exposure of (002) facet. The 2D ZIF8 possessed the BET surface area of $1633 \mathrm{~m}^{2} / \mathrm{g}$ and the pore diameter size of $0.63 \mathrm{~nm}$ (Fig. 4e).
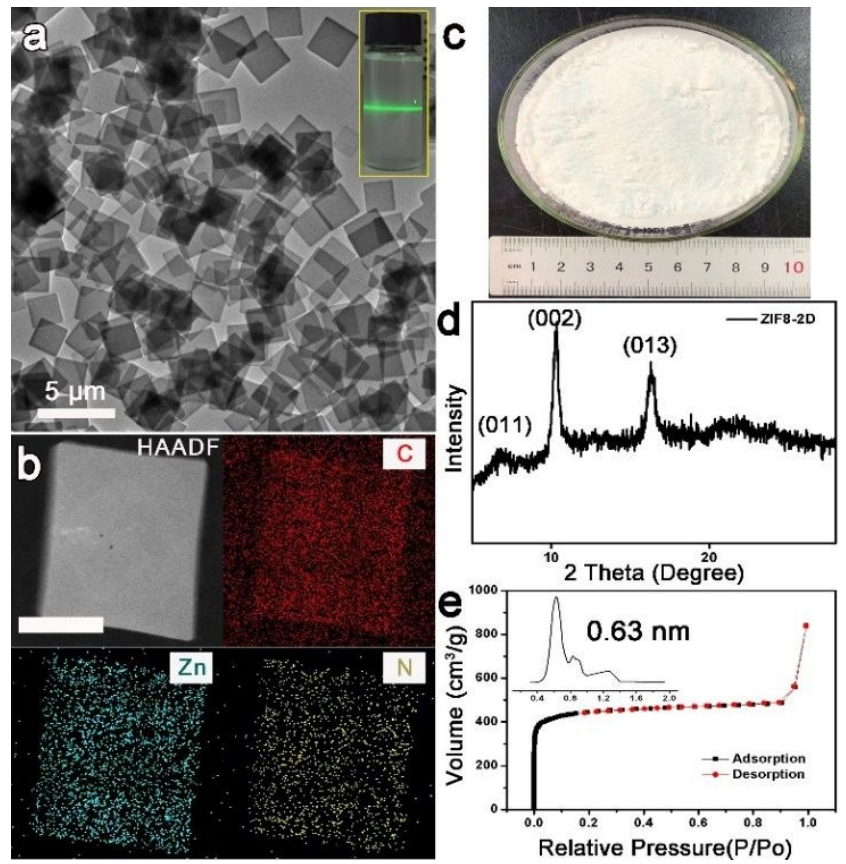

Fig. 4 (a) TEM image of 2D ZIF-8 (002). (b) Elemental mapping of 2D ZIF-8 (002), showing the distribution of C (red), Zn (blue), N (yellow). (c) the mass production of as-prepared 2D ZIF-8 (002). (d) XRD pattern of 2D ZIF-8 (002). (e) $\mathrm{N}_{2}$ adsorption/desorption isotherms, and pore size distributions of 2D ZIF-8 (002).

In summary, we presented a new concept to realize the facet engineering in MOF materials by shaping them into $2 \mathrm{D}$ nanostructures. ZIF-67 and ZIF-8 have been successfully synthesized with dominant exposure of (002) facets. By OER test, we verified the facet effect on catalytic performance with the ZIF-67 (002), which demonstrated greatly superior OER properties to size-comparable ZIF-67 nanocrystals as well as commercial $\mathrm{IrO}_{2}$. DFT calculations also proved the lower overpotential of ZIF-67 (002) than (011) and (111) facets. We hope this facet engineering approach will promote the development of MOF materials in broad applications.

This work was supported by the National Key R\&D Program of China (2018YFA0702003) and the National Natural Science Foundation of China (21890383, 21671117, 21871159, 21901135, 51802306).

\section{Notes and references}

1 W. Ye, Z. T. Sun, C. M. Wang, M. S. Ye, C. H. Ren, R. Long, X S. Zheng, J. F. Zhu, X. J. Wu and Y. J. Xiong, Nano Res., 2018, 11, 3313-3326
2 L. Liu, Y. Jiang, H. Zhao, J. Chen, J. Cheng, K. Yang and Y. Li ACS Catalysis, 2016, 6, 1097-1108. DOl: 101039/DoCC00700E

3 G. Zhou, L. Jiang and D. He, J. Catal., 2019, 369, 352-362.

4 H. Robatjazi, D. Weinberg, D. F. Swearer, C. Jacobson, M. Zhang, S. Tian, L. Zhou, P. Nordlander and N. J. Halas, Sci. Adv. 2019, 5, eaav5340.

5 M. Yaghi, G. Li and H. Li, Nature, 1995, 378, 703-706.

6 S. Yuan, L. Feng, K. C. Wang, J. D. Pang, M. Bosch, C. Lollar, Y. Sun, J. Qin, X. Yang, P. Zhang, Q. Wang, L. Zou, Y. Zhang, L. Zhang, Y. Fang, J. Li and H.-C. Zhou, Adv. Mater., 2018, 30, 1704303.

7 T. Drake, P. Ji and W. Lin, Acc. Chem. Res., 2018, 51, 2129 2138.

8 K. Shen, L. Zhang, X. Chen, L. Liu, D. Zhang, Y. Han, J. Chen, J. Long, R. Luque, Y. Li and B. Chen, Science, 2018, 359, 206-210.

9 D. Li, S. Yu and H. Jiang, Adv. Mater., 2018, 30, 1707377.

10 M. T. Zhao, K. Yuan, Y. Wang, G. Li, J. Guo, L. Gu, W. Hu, H. Zhao and Z. Y. Tang, Nature, 2016, 539, 76-80.

11 Y. Pi, X. Li, Q. Xia, J. Wu, Z. Li, Y. Li and J. Xiao, Nano Res., 2017, 10, 3543-3556.

12 S. Bai, X. Liu, K. Zhu, S. Wu and H. Zhou, Nat. Energy, 2016, 1 16094.

13 R. W. Flaig, T. M. Osborn Popp, A. M. Fracaroli, E. A. Kapustin, M. J. Kalmutzki, R. M. Altamimi, F. Fathieh, J. A. Reimer and O. M. Yaghi, J. Am. Chem. Soc., 2017, 139, 12125-12128.

14 T. Rodenas, I. Luz, G. Prieto, B. Seoane, H. Miro, A. Corma, F. Kapteijn, F. X. Llabrés i Xamena and J. Gascon, Nat. Mater., 2015, 14, 48-55.

15 D. Liu, G. Pang, Z. Tang and S. Feng, Inorg. Chem. Front., 2019, 6, 2043-2049.

16 H. Furukawa, K. E. Cordova, M. O'Keeffe and O. M. Yaghi, Science, 2013, 341, 1230444.

17 F.-L. Li, P. Wang, X. Huang, D. J. Young, H.-F. Wang, P. Braunstein and J.-P. Lang, Angew. Chem. Int. Ed., 2019, 131, 7125-7130.

18 M. Zhao, Y. Huang, Y. Peng, Z. Huang, Q. Ma and H. Zhang, Chem. Soc. Rev., 2018, 47, 6267-6295.

19 H. Kim, S. Yang, S. R. Rao, S. Narayanan, E. A. Kapustin, H. Furukawa, A. S. Umans, O. M. Yaghi and E. N. Wang, Science, 2017, 356, 430-434.

20 J. Q. Shen, P. Q. Liao, D. D. Zhou, C. T. He, J. X. Wu, W. X Zhang, J. P. Zhang and X. M. Chen, J. Am. Chem. Soc., 2017, 139, 1778-1781.

21 G. Hai, X. Jia, K. Zhang, X. Liu, Z. Wu and G. Wang, Nano Energy, 2018, 44, 345-352.

22 S. Zhao, Y. Wang, J. Dong, C. T. He, H. Yin, P. An, K. Zhao, X. Zhang, C. Gao, L. Zhang, J. Lv, J. Wang, J. Zhang, A. Muqsit Khattak, N. Ali Khan, Z. Wei, J. Zhang, S. Liu, H. Zhao and Z. Tang, Nat. Energy, 2016, 1, 16184.

23 M. Liu, L. Kong, X. Wang, J. He and X.-H. Bu, Small, 2019, 15 1903410.

24 J. Zhang, M. Chen, J. Chen, H. Li, S. Wang, Q. Kuang, Z. Cao and Z. Xie, Sci. China Mater., 2017, 60, 685-696.

25 W. Liao and G. Zhou, Sci. China Mater., 2017, 60, 664-673.

26 J. Wu, Z. Ren, S. Du, L. Kong, B. Liu, W. Xi, J. Zhu and H. Fu, Nano Res., 2016, 9, 713-725.

27 X.-R. Wang, J.-Y. Liu, Z.-W. Liu, W.-C. Wang, J. Luo, X.-P Han, X.-W. Du, S.-Z. Qiao and J. Yang, Adv. Mater., 2018, 30, 1800005.

28 H. Andersen, K. Xu, D. Malyshkin, R. Strandbakke and A. Chatzitakis, Chem. Commun., 2020, 56, 1030-1033.

29 J. Rossmeisl, Z. W. Qu, H. Zhu, G. J. Kroes and J. K. Nørskov, J. Electroanal. Chem., 2007, 607, 83-89.

30 I. C. Man, H. Su, F. Calle-Vallejo, H. Hansen, J. Martínez, N Inoglu, J. Kitchin, T. Jaramillo, J. K. Nørskov and J. Rossmeisl, ChemCatChem 2011, 3, 1159 


\section{Table of Contents}

We present a facile and low-cost method to shape ZIF into 2D nanosheets with exposed (002) facets and discover that they exhibit excellent activity for oxygen evolution reaction.

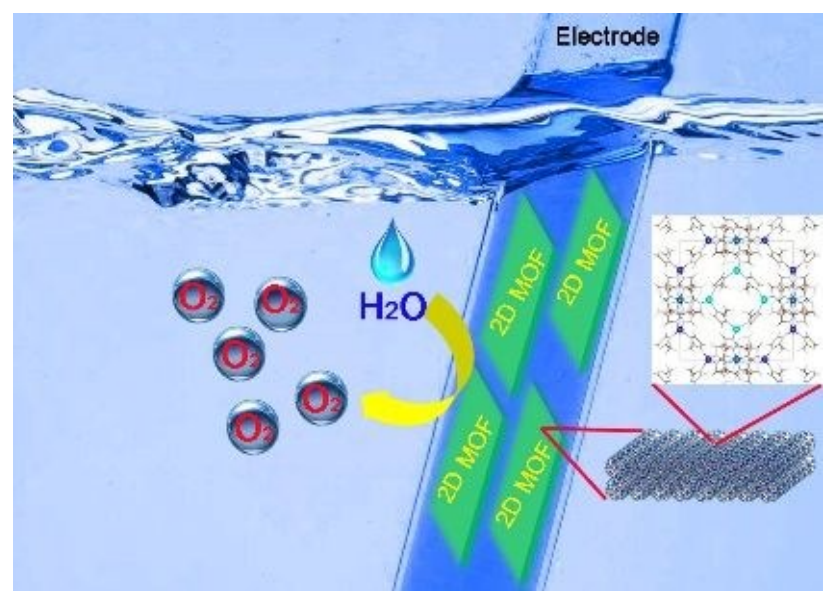

